\title{
A Modified Enhanced Recovery after Surgery (ERAS): Use and Surgical Outcome in Breast Cancer Patients
}

\author{
Tajudeen A. Wahab*, Hanna Uwakwe, Maher Jumah, Rilwan Aransi, Humayun Kabir Khan \\ Elm Breast Care Centre, Barking, Havering and Redbridge University Hospitals NHS Trust, King George Hospital, London, UK \\ Email: *oroki40@yahoo.com
}

How to cite this paper: Wahab, T.A., Uwakwe, H., Jumah, M., Aransi, R. and Khan, H.K. (2018) A Modified Enhanced Recovery after Surgery (ERAS): Use and Surgical Outcome in Breast Cancer Patients. Journal of Biosciences and Medicines, 6, 15-25.

https://doi.org/10.4236/jbm.2018.63002

Received: February 6, 2018

Accepted: March 11, 2018

Published: March 14, 2018

Copyright $\odot 2018$ by authors and Scientific Research Publishing Inc. This work is licensed under the Creative Commons Attribution International License (CC BY 4.0).

http://creativecommons.org/licenses/by/4.0/

(c) (i) Open Access

\begin{abstract}
Background: ERAS protocols are perioperative interventions aimed at reducing postoperative complications, length of hospital stay (LOS) and early return to normal activities. This has improved outcome in many surgical specialties, including breast surgery. We present the surgical outcome of breast cancer (BC) patients treated over a 12-month period following the principle of ERAS protocols and highlight the underpinning evidence. Methods: A retrospective analysis of all BC patients diagnosed and had breast cancer surgery over 12 months. Data collected included patient's demographics, type of surgery, LOS, other perioperative care and significant postoperative complications. Excluded were patients with bilateral cancer surgeries, diagnostic excision, margin clearance or breast reconstruction. Results: There were $621 \mathrm{BC}$ diagnosed including 5 male and 12 bilateral female BC. The ages ranged from 25 to 93 years. Excluding bilateral BC, 351 patients (70.2\%) had breast conserving surgery (BCS) while $149(29.8 \%)$ patients had mastectomy as index cancer surgery. Sixteen (4.5\%) of the women who initially underwent BCS subsequently had a completion mastectomy. The overall rate of successful BCS was 335/500 (67\%). 441 (85.5\%) of patients were discharged same or next day. $12(7.2 \%)$ cases of postoperative haematoma, 6 cases of wound infection and a case of seroma requiring surgical/radiological drainage recorded. Conclusion: ERAS protocol in BC surgery is associated with decreased LOS and low complication rate. Delayed discharges are mostly due to adverse social factors and medical comorbidity rather than post-operative surgical complications.
\end{abstract}

\section{Keywords}

ERAS $^{\circledR}$, Breast Cancer, Surgical Outcome 


\section{Introduction}

Fast track surgery, also known as "enhanced recovery after surgery" (ERAS), was pioneered by Henrik Kehlet, a Danish colorectal surgeon [1]. It is evidence based multidisciplinary, multi-modal approach to improving perioperative care and mitigates the adverse effect of perioperative stress arising from major surgery. The pathways aim to maintain physiological function, early mobilisation, pain relief and commencement of early oral nutrition. ERAS protocol has been validated in many surgical specialties, including colorectal surgery [2], urology [3] and gynaecology [4]. Arsalani-Zadeh, et al. in a review of literature suggested that the principles of enhanced recovery can be adopted in breast cancer surgery and have proposed a 12-point protocol for a prospective evaluation (Figure 1) [5].

Initiation of an ERAS pathway in microvascular breast reconstruction has resulted in a significant decreased hospital LOS and the amount of postoperative opioids use without increase in patient-reported pain [6].

Our breast unit had adopted the pathway of "day case/one night stay" for breast surgery incorporating the principles of ERAS as routine. The aim of this study is to present the surgical outcome of our BC patients treated over a 12-month

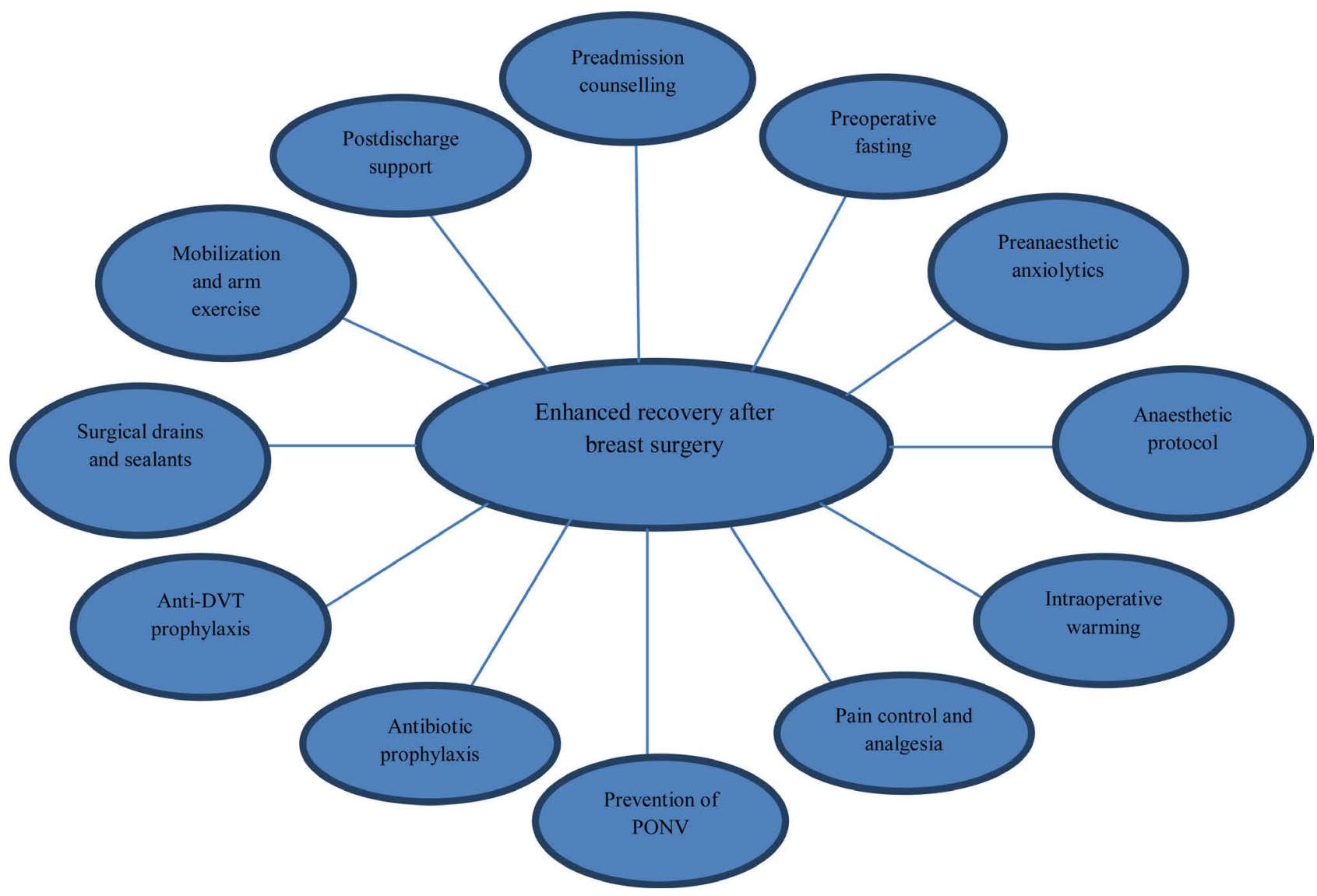

(Reproduced with authors' permission).

Figure 1. Schematic representation of enhanced recovery after surgery for breast cancer. DVT, deep vein thrombosis; PONV, postoperative nausea and vomiting [5]. 
period in terms of the LOS, significant post-operative complications and a review of the evidence underpinning our practice.

\section{Material and Methods}

A retrospective analysis of all breast cancer patients diagnosed in our institution and had breast cancer surgery between April 2015 and March 2016 was performed. Data were collected from the theatre electronic patients' record, medical charts, electronic clinic and discharge letters and record kept by the specialist breast care nurses. Data collected included the patients' demographics, LOS, type of surgery, postoperative analgesia, use of prophylactic antibiotic and low suction drain, and significant postoperative complications like haematomas, seroma, wound infections needing active intervention and readmissions. This was entered into a spreadsheet for analysis. We excluded patients that had bilateral breast surgery, diagnostic excision, further margin excision for clearance, any form of breast reconstruction in the final analysis with regard to the LOS and significant complications. Minor complications that might have been treated by the district Nurse or the GP and does not require an acute visit to the hospital or readmission were excluded from the analysis.

\section{Results}

Six hundred and twenty one cases of BC were diagnosed within the 12 months period. This includes 5 male and 12 bilateral female breast cancers. The ages ranged from 25 to 93 years with most patients in age group 61 - 70 years (Figure 2).

The patients' American Association of Anaesthesiologist (ASA) [7] physical grading before surgery is as in Figure 3 .

The patients were operated on by or under the supervision of one of the 5 specialist breast surgeons. With the exclusion of bilateral breast cancers, 351

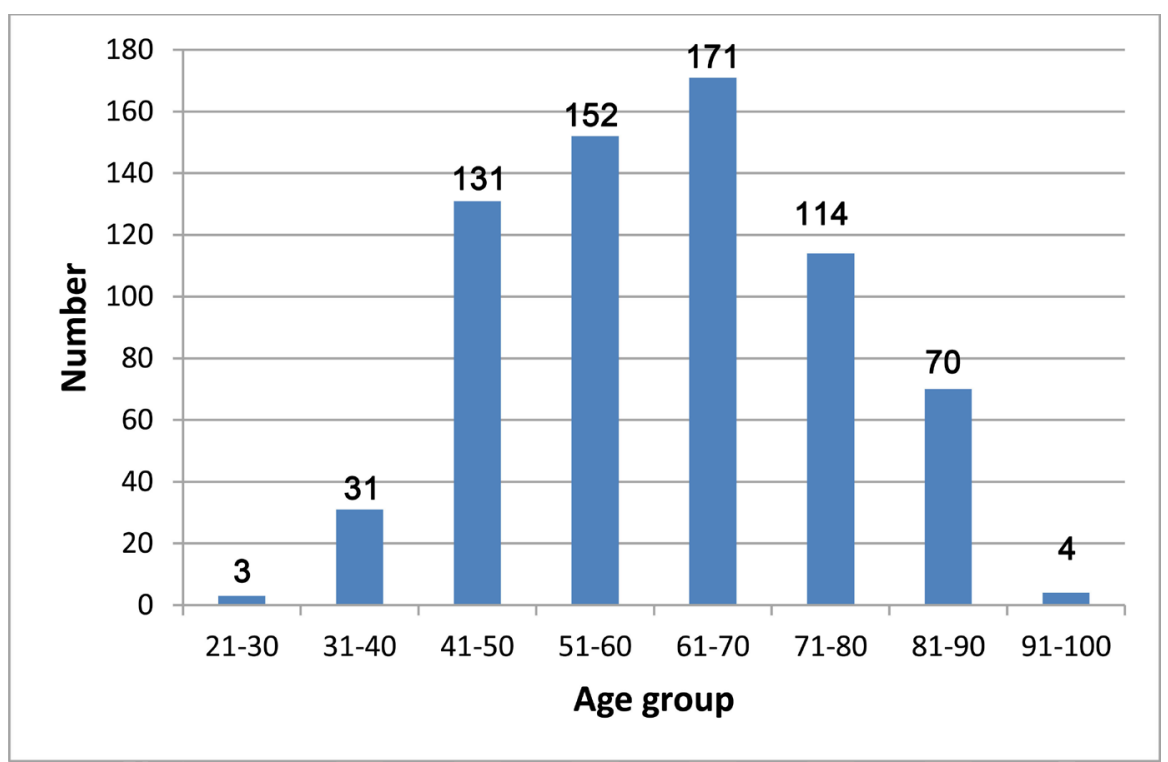

Figure 2. Age distribution of breast cancer patients that had surgery. 


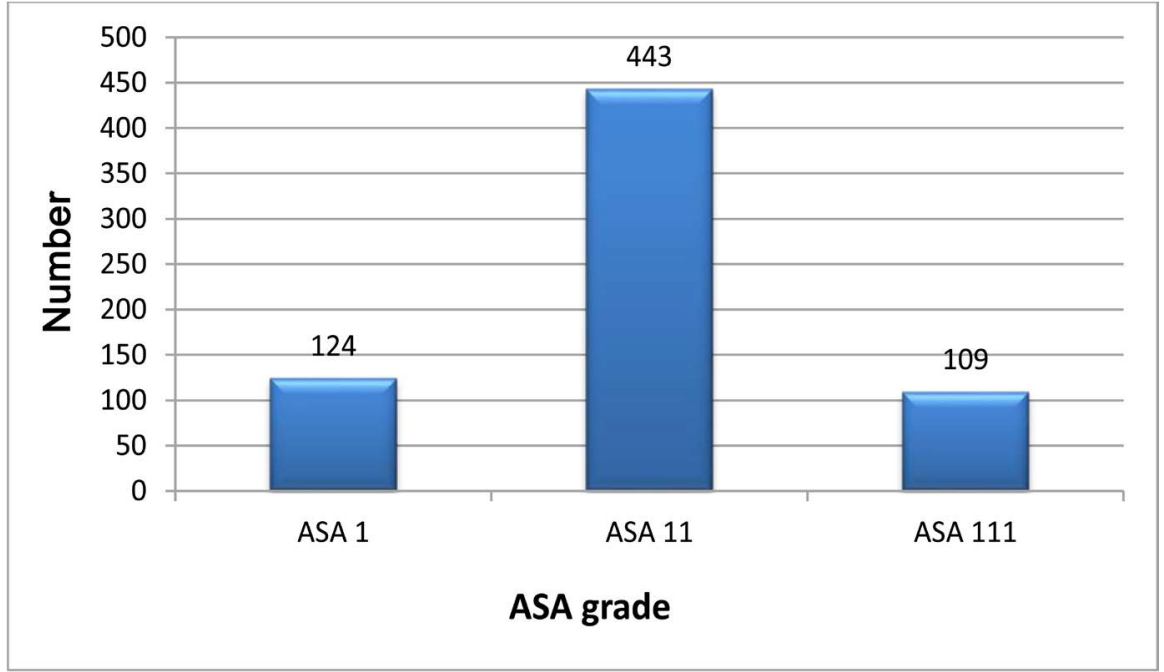

Figure 3. American Association of Anaesthesiologist (ASA) [7] grade.

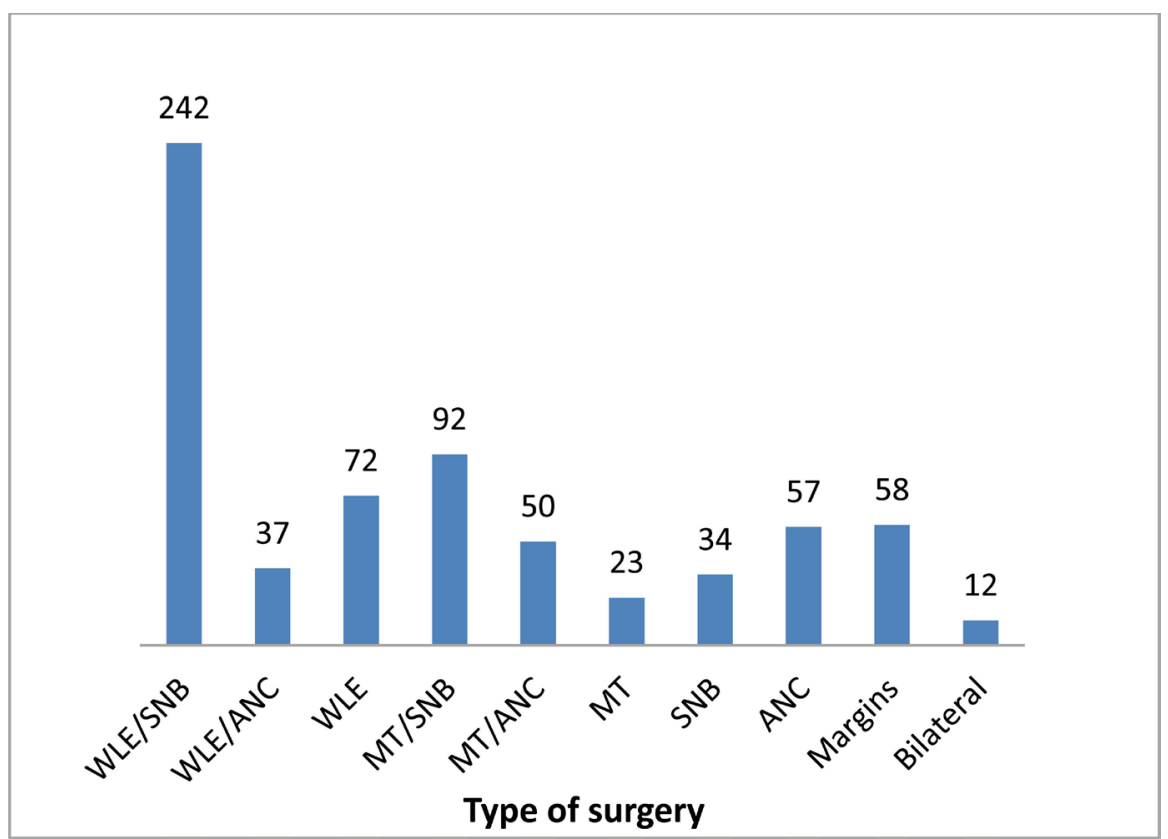

Figure 4. Type of surgery. WLE: wide local excision; SNB: sentinel node biopsy; ANC: axillary nodes clearance; MT: mastectomy.

patients $(70.2 \%)$ had breast conserving surgery (BCS) and 149 (29.8\%) patients had mastectomy as their index cancer surgery (Figure 4).

Sixteen (4.5\%) of the women who initially underwent BCS subsequently had a completion mastectomy. The overall rate of successful BCS was 335/500 (67\%). Majority $(441 ; 85.5 \%)$ of patients that had BCS \pm mastectomy \pm axillary surgery went home on the same or next day (Figure 5).

The majority of delayed discharges beyond a night stay were due to social reasons and pre-existing medical co-morbidity that needed stabilisation rather than post-operative surgical complications (Table 1 ).

There were $12(7.2 \%)$ cases of postoperative haematoma, 6 cases of wound 


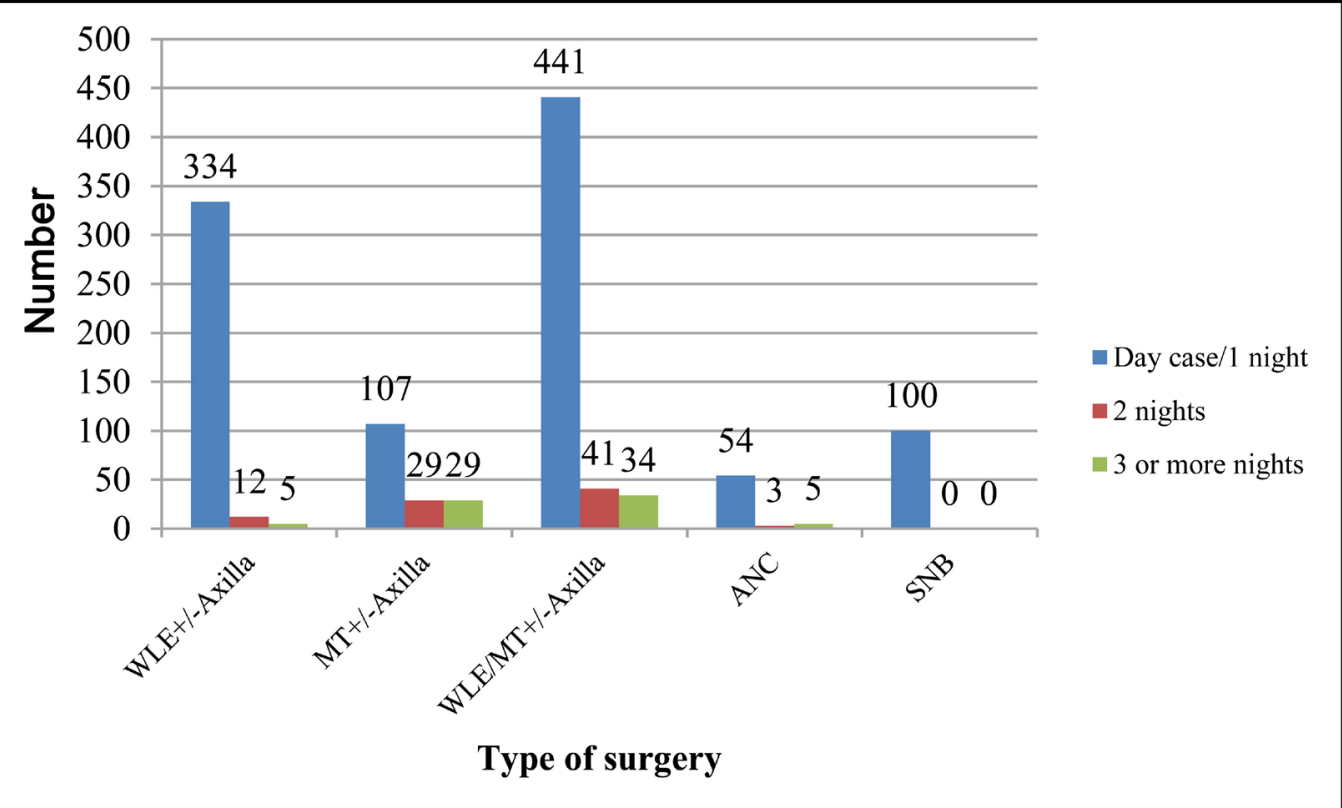

Figure 5. Length of hospital stay for different types of surgery.

Table 1. Causes of delayed discharge beyond one night hospital stay.

\begin{tabular}{cc}
\hline Cause of delayed discharge & Number \\
\hline Significant pre-existing comorbidity & 18 \\
Acute post-operative medical complication & 7 \\
Postoperative haematoma & 12 \\
Lack of support at home & 24 \\
Frailty & 8 \\
Patient refusing discharge & 3 \\
Not stated & 6 \\
\hline
\end{tabular}

infection and a case of seroma that required surgical/radiological drainage recorded.

\section{Discussion}

Traditional treatment for breast cancer which consisted of a modified radical mastectomy and axillary nodes clearance still remain the main mode of surgical treatment for breast cancer in many developing countries [8] [9]. This has been attributed to the fact that many of the patients are relatively young, presenting with a larger and more aggressive tumour at a more advanced stage [8] [10], coupled with lack of adequate facilities for radiotherapy and post-operative follow up in the developing countries [9]. Post operatively, most patients were kept on admission for up to 9 - 15 days and wound drains are removed before they are discharged [9]. This is in contrast to most developed countries where greater breast cancer awareness and adequate screening programme mean that most breast cancers are diagnosed at an earlier stage. Most breast cancers are thus 
amenable to breast conserving surgery (BCS), usually a wide local excision (WLE) with or without a sentinel node biopsy (SNB) or axillary nodes clearance (ANC). For instance, out of nearly 55,000 breast cancer diagnosed in the UK in 2014, 76\% were in stages $1 \& 2,13 \%$ stages $3 \& 4$ while the rest $11 \%$ have their stages undocumented at time of diagnosis. Demographically, almost half (48\%) of cases were diagnosed in people aged 65 and over [11]. Over a 12 months study period in our institution, which is among the larger breast units in the country, there were 612 cases of breast cancer (screen detected and symptomatic) diagnosed with 1028 breasts cancer surgery performed (mastectomy, wide local excision, axillary nodes clearance and sentinel nodes biopsy).

Randomised controlled trial (RCT) that compared discharge from hospital 4 days after surgery (with drain in situ) with discharge after drain removal (mean 9 days in hospital) found no significant differences in wound complications, duration of drainage, patient satisfaction, or psychosocial outcomes [12]. A similar conclusion that discharge two days after surgery (with drain in situ) compared with standard management (discharge after removal of drain) did not result in increased rates of physical or psychological illness [13]. A decade later, studies have demonstrated that with appropriate selections that exclude patients with high-risk comorbidities and concurrent surgery, it is safe to perform major breast cancer surgery on a same-day basis [14]. In a systematic review of eleven studies of moderate quality that compared day case breast surgery with in -patient care, the rate of discharge after day surgery was high, ranging from 86 to $100 \%$. The acute re-admission rates ranged from $0 \%$ to $7 \%$ with day surgery and a re-operation rates of between $14 \%$ to $40 \%$ [15].

As demonstrated from our results, despite the fact that majority of patients were in ASA group 2 and significant number in group 3, most of our patients are discharged next day after surgery. This was achieved by employing the principles of ERAS as highlighted below.

\subsection{Preadmission Counselling and Preoperative Fasting}

Each patient is looked after by a specialist breast care $(\mathrm{BCN})$ who delivered detailed preoperative information and counselling to patients and relatives. This includes the practicalities of each perioperative procedure, details of the operation, expected LOS for that procedure, drain and its management, patient's role and other information that may be peculiar to each patient. This is supplemented with various information leaflets. The presence of a clinical nurse specialist that explains the expectations during hospital admission result in greater adherence to the care pathway. This allows early recovery and shortening of hospital stay [16]. Patients attend a pre-assessment clinic where significant medical risk factors are identified and patient's condition is optimised. Patients are admitted on the day of surgery having fasted for no more than $2 \mathrm{~h}$ for liquids and $6 \mathrm{~h}$ for solid food in line with the recommendations of the European Society of Anaesthesiology [17]. We do not preload breast surgery patients with carbo- 
hydrate drinks as obtained in ERAS protocols for digestive surgery. This is because breast operations are generally relatively short procedures with no significant fluid shift, ileus or post-operative events that may delay oral intake.

\subsubsection{Preanaesthetic Anxiolytics, Anaesthetic Protocol and Intraoperative Warming}

The anaesthetists employ techniques that ensure minimum stress and maximum comfort for the patients allowing for a rapid recovery and early discharge. Essential routine medication is allowed in the morning of surgery and pharmacological premedication are rarely prescribed. Anxiolytic premedication has been shown to slightly delay recovery from anaesthesia [18]. The anaesthetic protocol consist mainly of routine use of laryngeal mask and occasional endotracheal intubation, propofol for induction and maintenance of anaesthesia, Fentanyl in repeated doses depending on the duration of the surgery, I.V paracetamol and parecoxib. Intraoperative intravenous fluid is restricted to maintaining euvolaemia and avoiding intraoperative fluid overload. Wounds are routinely infiltrated with bupivacaine. We do not employ regional paravertebral, epidural, or subpectoral blocks as part of analgesia/anaesthesia as there is no enough evidence to recommend their use [5]. An intraoperative warming blanket is used to maintain a perioperative normothermia which reduces surgical site infection (SSI) and bleeding [19].

\subsubsection{Pain Control and Prevention of PONV}

Intractable vomiting, patient anxiety and pain control issues have been identified as main reasons for failing early discharge following breast surgery [15]. Our anaesthetists employed a multimodal approach to PONV. This includes routine prophylactic administration of dexamethasone (except in diabetics) and ondansetron intraoperative to which cyclizine is added in the recovery if needed. Postoperative analgesia consists mostly of paracetamol and NSAID with minimal use of opioid.

\subsubsection{Antibiotic Prophylaxis}

Breast surgery is classified as a "clean" surgery with the rate of SSI expected to be below $2 \%$ but in reality ranges from $3 \%-15 \%$ [20] [21]. A systematic review demonstrated that prophylactic antibiotics administered preoperatively significantly reduce the incidence of SSI in BC surgery when compared with placebo [22]. This is in contrast to the findings in a retrospective observational study that suggest that a single dose of preoperative antibiotic was not associated with a lower SSI rate of elective, non-reconstructive breast operations [23]. In our practice, we administer a single dose prophylactic intravenous antibiotic before skin incision only in selected cases identified as high risk for SSI. This includes patients that had neo-adjuvant chemotherapy, diabetes, high BM1 or undergoing a reoperation.

\subsubsection{DVT Prophylaxis}

LMWH or unfragmented heparin to reduce the risk of thromboembolic (TE) 
complication is used selectively and limited to patients with high risk of TE, with the dosage and duration of treatment individualised based on the calculated risk of DVT. It is given a day after the operation in surgery involving mastectomy and ANC where the risk of post-operative haematoma is higher, otherwise 6 hours after surgery for day cases. TEDs stocking are used routinely and intermittent pneumatic compression stockings added if necessary. None of our patients developed a symptomatic DVT the incidence of which in a retrospective review was a low as $0.16 \%$ when early ambulation and mechanical antithrombotic measures are used routinely [24].

\subsubsection{Surgical Drains and Sealants}

We use diathermy for mastectomy flap dissection and wide local excision which we believe contributed to the low incidence of haematoma in our study. We routinely use of a low suction drains in patients who had mastectomy and axillary nodes clearance. The drains are managed in the community by the district nurse who is in touch with the breast team in case of any issue. Drains are removed when less than $50 \mathrm{ml} / 24$ hour or on the $5^{\text {th }}$ postoperative day. In two systematic reviews of RCT studies comparing drain or no drain after axillary dissection for $\mathrm{BC}$, there was reduced incidence of and number of post-operative seroma aspirations when drains were inserted with no significant difference in infection rates in the two groups [25] [26]. This is in contrast to the evidence from other two studies [27] [28] that showed no significant difference in seroma rate or complications if a wound drain was not used.

\subsection{Mobilization, Arm Exercise and Post-Discharge Support}

Patients return to normal diet and mobilise as soon as possible once recovered from the anaesthesia. The physiotherapist review and design an exercise program for each patient which is commenced before discharge. Early rather than delayed onset of training is valuable in avoiding deterioration in range of shoulder movement with no adverse impact on the incidence of postoperative lymphedema [29]. The wound dressings used are mostly skin glue or waterproof, allowing patients to wash and shower as normal postoperatively. Discharged patients are contacted a day after by the BCN for a telephone follow-up advice and support. The district nursing team also contact patients a day after discharge to check on the wound and the drain. They are reviewed in the result clinic 3 weeks after surgery for wound review and referral to oncologist for other adjuvant treatment as necessary.

\section{Conclusion}

The use of evidence-based enhanced recovery protocol in breast cancer surgery is associated with decreased length of hospital stay without increased complication rate. Most causes of delayed discharges are due to presence of adverse social factors and medical comorbidity rather than post-operative surgical complica- 
tions. We are driving improvement by promoting more of nurse led discharge for admitted patients, develop and pilot pathway of no drains and day case mastectomy for suitable patient and operate a smarter operation list. This may include operating BCS without ANC in the mornings or early on the afternoon list.

\section{Acknowledgements}

Thanks to our data coordinator, Albena Naydenova for her assistance in collection of data.

\section{Declaration of Conflicting Interests}

The authors declare that there is no conflict of interest.

\section{Funding}

This research received no specific grant from any funding agency in the public, commercial, or not-for-profit sectors.

\section{Ethical Approval}

None required.

\section{Informed Consent}

Not required.

\section{Authors Contributions}

TAW, HW, MJ, RA and HK made a substantial contribution to the concept and design, acquisition, analysis and interpretation of data, drafting and critical revision of the article for important intellectual content and approved the version to be published.

\section{Declaration}

We, the under listed authors, give the warranty that we are submitting our original work, that we have the rights in the work, that we are submitting the work for first publication in your Journal and that it is not being considered for publication elsewhere and has not already been published elsewhere, and that we have obtained and can supply all necessary permissions for the reproduction of any copyright works not owned by us.

\section{References}

[1] Kehlet, H. (1997) Multimodal Approach to Control Postoperative Pathophysiology and Rehabilitation. British Journal of Anaesthesia, 78, 606-617. https://doi.org/10.1093/bja/78.5.606

[2] Gustafsson, U.O., Scott, M.J., Schwenk, W., et al. (2013) Guidelines for Perioperative Care in Elective Colonic Surgery: Enhanced Recovery After Surgery (ERAS ${ }^{\circledast}$ ) 
Society Recommendations. World Journal of Surgery, 37, 259-284.

https://doi.org/10.1007/s00268-012-1772-0

[3] Enhanced Recovery Programme-British Association of Urological Surgeons. http://www.baus.org.uk/professionals/baus_business/.../enhanced_recovery_progra $\underline{\mathrm{mme}}$

[4] Nelson, G., Altman, A., Nick, A., et al. (2015) Guidelines for Pre- and Intra-Operative Care in Gynaecologic/Oncology Surgery: Enhanced Recovery after Surgery (ERAS ${ }^{\circledast}$ ) Society Recommendations, Gynecologic Oncology, 140, 313-322. http://doi.org/10.1016/j.ygyno.2015.11.015

[5] Arsalani-Zadeh, R., ElFadl, D., Yassin, N., et al. (2011) Evidence-Based Review of Enhancing Postoperative Recovery after Breast Surgery. Br J Surg., 98, 181-196. https://doi.org/10.1002/bjs.7331

[6] Batdorf, N.J., Lemaine, V., Lovely, J.K., et al. (2015) Enhanced Recovery after Surgery in Microvascular Breast Reconstruction. J Plast Reconstr Aesthet Surg., 68, 395-402. https://doi.org/10.1016/j.bjps.2014.11.014

[7] ASA Physical Status Classification System. https://www.asahq.org/resources/clinical-information/asa-physical-status-classificat ion-system. Accessed on 14/02/2018

[8] Clegg-Lamptey, J. and Hodasi, W.M. (2007) A Study of Breast Cancer in Korle Bu Teaching Hospital: Assessing the Impact of Health Education. Ghana Medical Journal, 41, 72-77.

[9] Ogundiran, T.O., Ayandipo, O.O., Ademola, A.F., et al. (2013) Mastectomy for Management of Breast Cancer in Ibadan, Nigeria. BMC Surgery, 13, 59. https://doi.org/10.1186/1471-2482-13-59

[10] Adebamowo, C.A. and Ajayi, O.O. (2000) Breast Cancer in Nigeria. West Afr J Med., 19, 179-191.

[11] Cancer Research UK. http://www.cancerresearchuk.org/health-professional/cancer-statistics/statistics-bycancer-type/breast-cancer/incidence-invasive\#heading-Three

[12] Bonnema. J., van Wersch, A.M.E., van Geel, A.N., et al. (1998) Medical and Psychosocial Effects of Early Discharge after Surgery for Breast Cancer: Randomised trial. BMJ, 316, 1267-1271. https://doi.org/10.1136/bmj.316.7140.1267

[13] Bundred, N., Maguire, P., Reynolds, J., et al. (1998) Randomised Controlled Trial of Effects of Early Discharge after Surgery for Breast Cancer. BMJ, 317, 1275-1279. https://doi.org/10.1136/bmj.317.7168.1275

[14] Cordeiro, E., Jackson, T. and Cil, T. (2016) Same-Day Major Breast Cancer Surgery Is Safe: An Analysis of Short-Term Outcomes Using NSQIP Data. Annals of Surgical Oncology, 23, 2480-2486. https://doi.org/10.1245/s10434-016-5128-0

[15] Marla, S. and Stallard, S. (2009) Systematic Review of Day Surgery for Breast Cancer. International Journal of Surgery, 7, 318-323. https://doi.org/10.1016/j.ijsu.2009.04.015

[16] Forster, A.J., Clark, H.D., Menard, A., et al. (2005) Effect of a Nurse Team Coordinator on Outcomes for Hospitalized Medicine Patients. The American Journal of Medicine, 118, 1148-1153. https://doi.org/10.1016/j.amjmed.2005.04.019

[17] Smith, I., Kranke, P., Murat, I., et al. (2011) European Society of Anaesthesiology. Perioperative Fasting in Adults and Children: Guidelines from the European Society of Anaesthesiology. European Journal of Anaesthesiology, 28, 556-569. https://doi.org/10.1097/EJA.0b013e3283495bal 
[18] Sheen, M.J., Chang, F.L. and Ho, S.T. (2014) Anesthetic Premedication: New Horizons of an Old Practice. Acta Anaesthesiologica Taiwanica, 52, 134-142. https://doi.org/10.1016/j.aat.2014.08.001

[19] WHO Surgical Site Infection Prevention Guidelines. http://www.who.int/gpsc/appendix14.pdf

[20] Lefebvre, D., Penel, N., Deberles, M.F., et al. (2000) Incidence and Surgical Wound Infection Risk Factors in Breast Cancer Surgery. La Presse Médicale, 29, 1927-1932.

[21] Witt, A., Yavuz, D., Walchetseder, C., et al. (2003) Preoperative Core Needle Biopsy as an Independent Risk Factor for Wound Infection after Breast Surgery. Obstetrics and Gynecology, 101, 745-750.

[22] Jones, D.J., Bunn, F. and Bell-Syer, S.V. (2014) Prophylactic Antibiotics to Prevent Surgical Site Infection after Breast Cancer Surgery. Cochrane Database of Systematic Reviews, No. 3, CD005360. https://doi.org/10.1002/14651858.CD005360.pub4

[23] Crawford, C.B., Clay, J.A., Seydel, A.S., et al. (2016) Surgical Site Infections in Breast Surgery: The Use of Preoperative Antibiotics for Elective, Non-Reconstructive Procedures. International Journal of Breast Cancer, 2016, Article ID: 1645192. https://doi.org/10.1155/2016/1645192

[24] Andtbacka, R.H., Babiera, G., Singletary, S.E., et al. (2006) Incidence and Prevention of Venous Thromboembolism in Patients Undergoing Breast Cancer Surgery and Treated According to Clinical Pathways. Annals of Surgery, 243, 96-101. https://doi.org/10.1097/01.sla.0000193832.40178.0a

[25] Thomson, D.R., Sadideen, H. and Furniss, D. (2013) Wound Drainage after Axillary Dissection for Carcinoma of the Breast. The Cochrane Database of Systematic Reviews, 20, CD006823.

[26] He, X.D., Guo, Z.H., Tian, J.H., et al. (2011) Whether Drainage Should Be Used after Surgery for Breast Cancer? A Systematic Review of Randomized Controlled Trials. Medical Oncology, 28, S22-S30. https://doi.org/10.1007/s12032-010-9673-2

[27] NHS Improvement. Ambulatory Breast Surgical Care: Day Case and One Night Stay. http://www.evidence.nhs.uk/qipp

[28] Ebner, F.K., Friedl, T.W.P., deGregorio, N., et al. (2013) Does Non-Placement of a Drain in Breast Surgery Increase the Rate of Complications and Revisions? Geburtsh Frauenheilk, 73, 1128-1134. https://doi.org/10.1055/s-0033-1351071

[29] Chan, D.N., Lui, L.Y. and So, W.K. (2010) Effectiveness of Exercise Programmes on Shoulder Mobility and Lymphoedema after Axillary Lymph Node Dissection for Breast Cancer: Systematic Review. Journal of Advanced Nursing, 66, 1902-1914. https://doi.org/10.1111/j.1365-2648.2010.05374.x 